\title{
Sudden Onset Loss of Consciousness of a Multiple Cerebral Venous Sinuses Thrombosis Case, Accompanying With the Patient History of Dehydration and Fasting as Aggravating Factors: A Case Report and Literature Review
}

\author{
Arash Forouzan ${ }^{\mathrm{a}}$, Kambiz Masoumi ${ }^{\mathrm{a}}$, Hassan Motamed $^{\mathrm{a}}$, b, Mandana Ghanavati ${ }^{\mathrm{a}}$
}

\begin{abstract}
Cerebral venous sinus thrombosis (CVST) is a rare medical condition with probable devastating clinical consequences. It is a dilemma of clinical diagnosis because of its variable vague initial clinical symptoms. Sudden onset loss of consciousness is not an expectable initial clinical scenario. Here we report a 32-year-old female of Iranian ethnicity complaining of a short restricted vague headache episode, which started 1 hour before emergency department arrival. Shortly after arrival on emergency department, the patient complicated with sudden onset thunderclap form loss of consciousness accompanying with severe sweating. On investigation, diagnosis of multiple cerebral sinuses thrombosis was confirmed by magnetic resonance venography. For religious ceremonies, she has been fasting for several days before clinical symptoms were developed, and she has been also on oral contraceptive pills diet for a long time. Dehydration and fasting may be an aggravating predisposing factor with synergism effects. Possibly prolonged duration of dehydration due to fasting may have a significant role in mediating multiple CVST in our reported case. Due to multiple cerebral venous sinuses involvement, the mentioned case clinical scenario cannot be well matched with the persistent described clinical category structures of the literatures. This clinical symptom presentation form of sudden onset loss of consciousness is a more rare clinical manifestation of CVST. Complex vague different primary clinical presentation of CVST and its devastating probable clinical consequences necessitate more careful clinical vision in respect of correlated symptoms to rule out possible cerebral sinus thrombosis diagnosis. In this case report, we purpose to focus concentration on possible uncommon vague clinical presentation of CVST.
\end{abstract}

Keywords: Cerebral venous sinus thrombosis; Sudden onset loss of consciousness

\footnotetext{
Manuscript accepted for publication April 27, 2015

aEmergency Medicine Department, Ahvaz Jundishapur University of Medical Sciences, Ahvaz, Iran

bCorresponding Author: Hassan Motamed, Emergency Medicine Department, Ahvaz Jundishapur University of Medical Sciences, Ahvaz, Iran.

Email: hasan_motamed@yahoo.com
}

doi: http://dx.doi.org/10.14740/jnr340e

\section{Introduction}

Cerebral venous sinus thrombosis (CVST) is a rare medical condition with probable devastating clinical consequences with an estimated incidence of 5/1,000,000. It is culprit of approximately $0.5 \%$ of all strokes [1]. Women are affected three times more than men [2]. Superior sagittal sinus is most affected (70-80\%), then transverse, sigmoid, and in a lesser extent, cavernous sinus involvement is seen. Cortical and cerebellar veins may also be affected. One-third of cases have more than one sinus involvement [3]. The certain incidence of CVST is unknown, and it seems to be reported more in developing countries [4]. The CVST outcome may vary from full recovery to permanent neurologic sequel or death $[5,6]$. It is a dilemma of clinical diagnosis because of its variable vague initial clinical symptoms [7].

Predisposing factors which can accelerate occurrence of CVST consist of low cerebral blood flow, cranial tumors, penetrating head traumas, lumbar puncture, malignancy, inflammatory bowel disease, connective tissue disorders, Behcet's disease, sarcoidosis, nephrotic syndrome, pregnancy, puerperium, adjacent infectious processes, hypercoagulability, dehydration, oral contraceptives and hormone replacement therapy. In $20-25 \%$ of cases, the etiology cannot be determined [8-12].

In acute phase CVST, mortality rate reaches $4 \%$. One month period fatality predictors are depressed consciousness, mental state disorders, deep venous system thrombosis, right hemispheric hemorrhage and posterior fossa lesions. The most important etiology of acute death is transtentorial herniation [13]. Other causes of acute death include status epilepticus, pulmonary embolism and medical complications [14]. Diversity and vagueness of prodromal clinical findings in CVST make it more complex to diagnose. The more diagnosis and treatment delay occur, the more significant morbidity and mortality the patient will face.

Here we report a rare case of CVST with rapidly progressive decreasing loss of consciousness within minutes on presentation as its main first primary clinical manifestation. Such a rare symptom can be undiagnosed or misdiagnosed and lead to probable fatal/irreversible consequences due to delayed or mal-treatment. It is hoped that the current case presentation draws more concentration and attention of clinical practitioner to potentially rare non-discernible prodromal symptoms of

Articles $\left(\right.$ The authors | Journal compilation @ J Neurol Res and Elmer Press Inc ${ }^{\mathrm{TM}}$ | www.neurores.org

This is an open-access article distributed under the terms of the Creative Commons Attribution License, which permits unrestricted use, distribution, and reproduction 

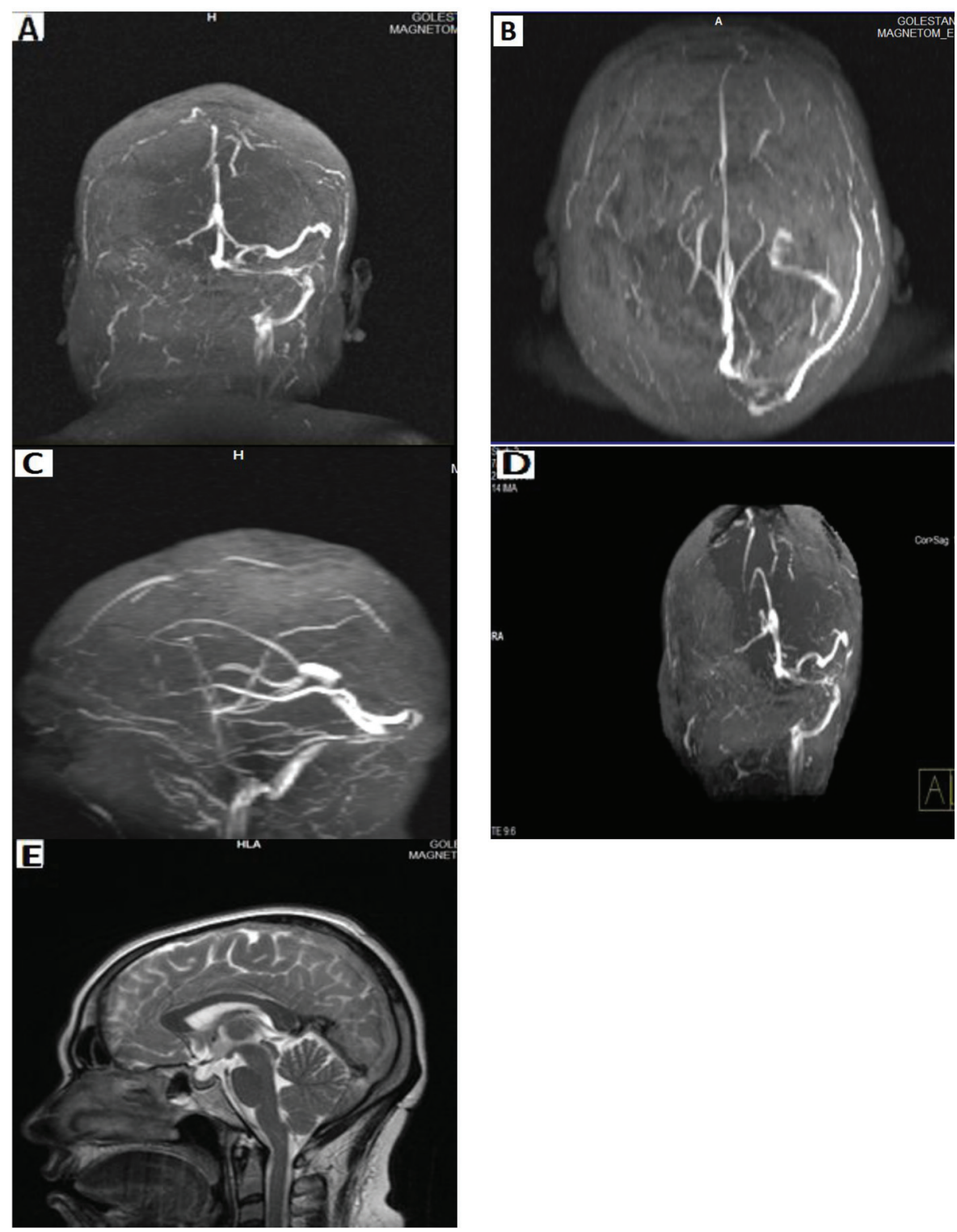

Figure 1. (A, B, C, D) Filling defects in sigmoid and transverse cerebral sinuses and internal jugular are suggesting of thrombosis. Partially involvement of superior sagittal sinus is remarkable. (E) Cord sign confirms superior sagittal sinus thrombosis.

possible serious devastating disease.

\section{Case Report}

A 32-year-old female of Iranian ethnicity complained of a short restricted vague mild-to-moderate non-pulsatile frontal headache episode, which started during a grief ceremony $1 \mathrm{~h}$ before emergency department arrival. The headache was nonpositional with a short duration of $4-5 \mathrm{~min}$, accompanying with nausea and two episodes of vomiting. The patient was oriented, and her speech was coherent with no delusion or hallucination.

Within 15 min after arrival, severe sweating and sudden loss of consciousness occurred. Examination revealed reac- tive myotic pupils. The patient was intubated for maintaining the airway. In suspicion of subarachnoid hemorrhage, lumbar puncture and analysis were performed which were normal. There was no visual or sensory motor deficit. On examination, extraocular eye movement and cranial nerves were intact and deep tendon reflexes were symmetrically normal. There was no chemosis and proptosis, and only on fundoscopy examination, a grade II papilledema, despite of normal disc and retina, was reported. In addition, normal cerebellar tests on arrival were evaluated. For religious ceremonies, she has been fasting for several days before clinical symptoms were developed, and she has been also on oral contraceptive pills diet for the last 7 years and 1 year history of intramuscular medroxy progesterone administration. She denied any positive associated past medical history. The patient underwent cranial computed 


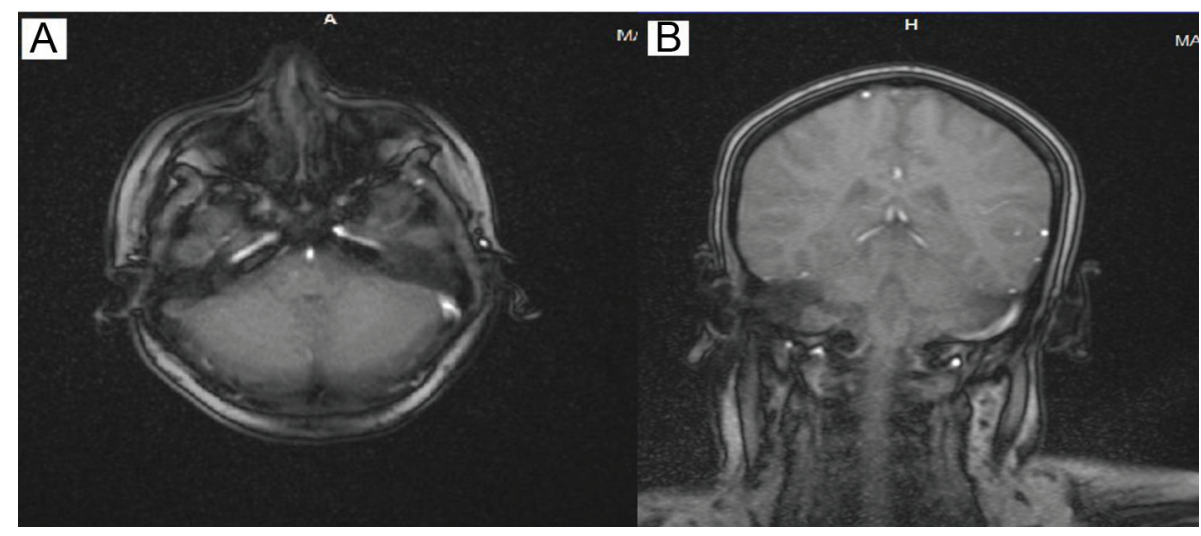

Figure 2. (A) Hypersignal area in right transverse sinus is seen. (B) Filling defect in MRI (T1) with contrast revealed findings compatible with right sigmoid sinus thrombosis.

tomography and magnetic resonance venography for more investigation. Magnetic resonance imaging and magnetic resonance venography revealed findings compatible with multiple CVST consisting of right sigmoid and transverse cerebral sinus and superior sagittal sinus (Fig. 1-3).

In the course of the patient observation and treatment, heparinization and anticoagulation with standard doses were applied. The level of consciousness was improved during the second day of admission, and headache was alleviated but papilledema was noted on examination. No neurological deficit developed during the hospitalization, and nausea and vomiting were subsided after 4 days. She was discharged with complete recovery without any additional signs and symptoms after 7 days of admission.

\section{Discussion}

Pattern and clinical pictures of CVST mainly are impressed by location and spread of affected venous territory with thrombosis. Clinical scenarios mainly are affected by the location of culprit sinus, patient age, presence of parenchymal lesions and time between the clinical presentation onset and the arrival to hospital [1]. The clinical scenarios mediate via two principal pathological mechanisms including localized edema of the brain and venous infarction and also intracranial hypertension. If large unilateral infarcts or hemorrhages compress the diencephalon and brain stem, patients may become comatose or die from cerebral herniation if untreated. Other causes of coma are involvement of thalamus and generalized seizure [15]. The most frequent clinical signs and symptoms consist of headache (95\%), focal seizure with or without generalization $(47 \%)$, unilateral and bilateral paresis (43\%) and papilledema (41\%) [5]. These clinical symptoms, signs and scenarios present in a wide range, but they can be generally categorized in four major syndromes, consisting of isolated intracranial hypertention, focal syndromes, encephalopathy and cavernous sinus syndromes. Less frequent symptoms are tinnitus and cranial nerve palsies [16].

Some other authors described these clinical category structures briefly different from the others, consisting of isolated intracranial hypertension, focal neurological abnormalities, seizures and encephalopathy. These syndromes may present in combination or isolation depending on the extent and location of cerebral venous thrombosis. Superior and inferior sagittal sinus thrombosis presents with motor deficit and seizure. Straight sinus thrombosis clinical scenario is motor deficit and mental status changes, despite of transverse sinus involvement which presents with intracranial hypertention (headache), tinnitus, cranial nerves palsies and aphasia (if left sided). Also

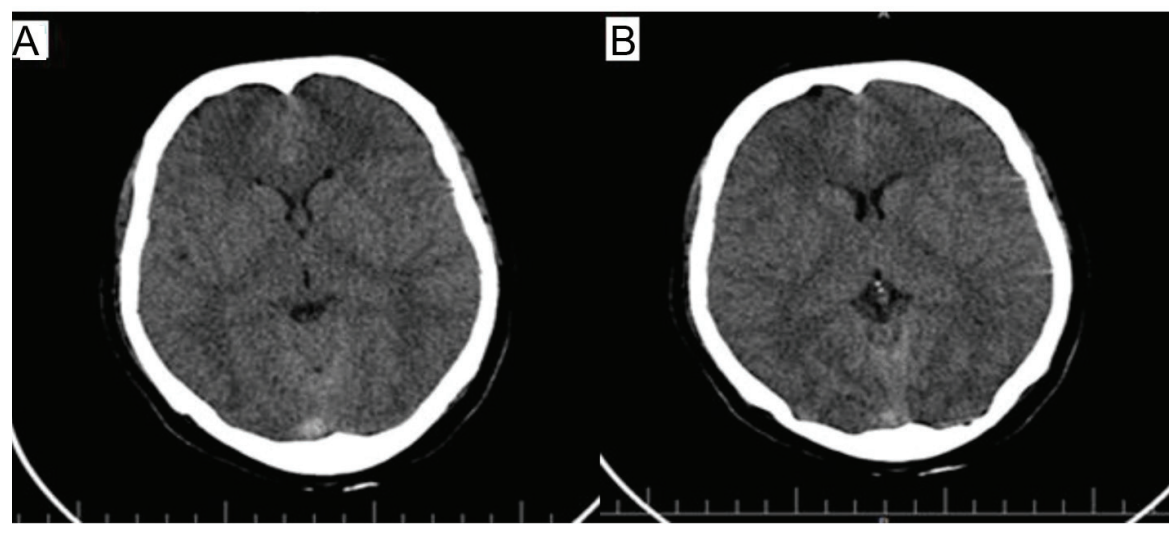

Figure 3. (A, B) CT scan showed no significant lesion. 
cavernous sinus thrombosis presentations are orbital pain, chemosis, proptosis and cranial nerve palsies (III-VI) [17].

In our case, sudden onset loss of consciousness occurred with a form of sympathetic tone overactivity. Multiple involvements of superior sagittal, transverse and sigmoid cerebral venous sinuses lead to thunderclap form sudden onset progression of loss of consciousness. Dehydration and fasting may be an aggravating predisposing factor with synergism effect in our case. Possibly prolonged duration of dehydration due to fasting may have a significant role in mediating multiple CVST in the reported case. Complex vague different primary clinical presentation of CVST and its devastating probable clinical consequences necessitate more careful clinical vision in respect of correlated symptoms to rule out possible cerebral sinus thrombosis diagnosis. To conclude, early diagnosis and treatment of CVST is crucial and multiple different factors may predispose.

\section{Acknowledgement}

We appreciate anyone who helped us on data gathering and report process.

\section{Conflict of Interest}

The authors state no conflict of interest.

\section{Abbreviations}

CVST: cerebral venous sinus thrombosis

\section{References}

1. Bousser MG, Ferro JM. Cerebral venous thrombosis: an update. Lancet Neurol. 2007;6(2):162-170.

2. Ferro JM, Canhao P, Stam J, Bousser MG, Barinagarrementeria F. Prognosis of cerebral vein and dural sinus thrombosis: results of the International Study on Cerebral Vein and Dural Sinus Thrombosis (ISCVT). Stroke. 2004;35(3):664-670.

3. Damak M, Crassard I, Wolff V, Bousser MG. Isolated lateral sinus thrombosis: a series of 62 patients. Stroke.
2009;40(2):476-481.

4. Kalita J, Bansal V, Misra UK, Phadke RV. Cerebral venous sinus thrombosis in a tertiary care setting in India. QJM. 2006;99(7):491-492.

5. de Bruijn SF, de Haan RJ, Stam J. Clinical features and prognostic factors of cerebral venous sinus thrombosis in a prospective series of 59 patients. For The Cerebral Venous Sinus Thrombosis Study Group. J Neurol Neurosurg Psychiatry. 2001;70(1):105-108.

6. Siddiqui FM, Kamal AK. Complications associated with cerebral venous thrombosis. J Pak Med Assoc. 2006;56(11):547-551.

7. Turkewitz LJ, Jacobs AK, Bidwell JK. Atypical MRI findings of venous sinus thrombosis in pregnancy: clinical significance relating to episodic vascular headache. Headache. 1991;31(4):240-243.

8. Cantu C, Barinagarrementeria F. Cerebral venous thrombosis associated with pregnancy and puerperium. Review of 67 cases. Stroke. 1993;24(12):1880-1884.

9. Lim YH. Aseptic cerebral venous sinus thrombosis in early pregnancy. Hong Kong J Emerg Med. 2007;14:237239.

10. Lemke DM, Hacein-Bey L. Cerebral venous sinus thrombosis. J Neurosci Nurs. 2005;37(5):258-264.

11. Ferro JM, Bacelar-Nicolau H, Rodrigues T, Bacelar-Nicolau L, Canhao P, Crassard I, Bousser MG, et al. Risk score to predict the outcome of patients with cerebral vein and dural sinus thrombosis. Cerebrovasc Dis. 2009;28(1):3944.

12. Ferro JM, Canhao P, Bousser MG, Stam J, Barinagarrementeria F. Cerebral vein and dural sinus thrombosis in elderly patients. Stroke. 2005;36(9):1927-1932.

13. Canhao P, Ferro JM, Lindgren AG, Bousser MG, Stam $\mathrm{J}$, Barinagarrementeria F. Causes and predictors of death in cerebral venous thrombosis. Stroke. 2005;36(8):17201725.

14. Diaz JM, Schiffman JS, Urban ES, Maccario M. Superior sagittal sinus thrombosis and pulmonary embolism: a syndrome rediscovered. Acta Neurol Scand. 1992;86(4):390396.

15. Stam J. Thrombosis of the cerebral veins and sinuses. N Engl J Med. 2005;352(17):1791-1798.

16. Ferro JM, Canhao P. Cerebral venous and dural sinus thrombosis. Practical Neurology. 2003; 3:214-219

17. Piazza G. Cerebral venous thrombosis. Circulation. 2012;125(13):1704-1709. 